\title{
Siringoma condroide en la mano. A propósito de un caso
}

\author{
V. García Virto ${ }^{(1)}$, C. Simón Pérez ${ }^{(2)}$, M. A. Martín Ferreroo ${ }^{(3)}$ \\ (1) FEA Servicio de Cirugía Ortopédica y TRaumatología. Complejo Asistencial de Segovia \\ (2) FEA Servicio de Cirugía Ortopédica y TRaumatología. Hospital Clínico Universitario de Valladolid \\ (3) Profesor Titular de la Universidad De Valladolid, Jefe de la Unidad de Mano. Servicio de Cirugía \\ Ortopédica y Traumatología. Hospital Clínico Universitario de Valladolid
}

\author{
Correspondencia: \\ Dra. Clarisa Simón Pérez \\ Hospital Clínico Universitario de Valladolid \\ Servicio de Traumatología y Cirugía Ortopédica \\ Avda. Ramón y Cajal, s/n \\ 47005 Valladolid \\ Teléfono: 983420000 ext: 276 \\ e-mail: simonclarisa@yahoo.es
}

\begin{abstract}
El siringoma condroide (SC) es un tumor muy infrecuente de origen epitelial, por lo general benigno. Clínicamente se presenta en la cabeza y cuello y se caracteriza por ser un tumor subcutáneo o intradérmico, de consistencia firme. El diagnóstico definitivo se establece mediante el estudio anatomopatológico. El tratamiento consiste en la exéresis quirúrgica. En el presente artículo, se presenta un nuevo caso de SC de la mano, de localización extraordinariamente infrecuente.
\end{abstract}

Palabras clave: siringoma, condroide, mano, hipotenar.
Chondroid syringoma (SC) is a very rare tumor of epithelial origin, usually benign. Clinically it presents in the head and neck and is characterized by subcutaneous or intradermal tumor, firm. The diagnosis is made by pathologic examination. Treatment consists of surgical excision. In this article, we present a new case of SC hand, extremely rare location.

Key words: syringoma, chondroid, hand, hypothenar.

\section{INTRODUCCIÓN}

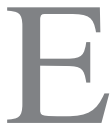
1 «tumor mixto» cutáneo fue descrito originalmente por Billroth ${ }^{1}$ en 1859 como una entidad nosológica con las mismas propiedades histopatológicas del tumor de la glándula salivar². El término «siringoma condroide» (SC) fue usado por primera vez en 1961 por Hirsch y Helwig para describir un tumor que presentaba estructuras propias de la glándula sudorípara en un estroma de aspecto cartilaginoso ${ }^{3}$. Finalmente Headington clasificó los siringomas condroides como ecrinos o apocrinos, en función de su apariencia histológica ${ }^{4}$.

De entre todos los tumores primarios cutáneos, el SC constituye un tumor raro con muy baja incidencia $(0,01-0,98 \%)$ y prevalencia ${ }^{5}$. Se trata de lesiones indolentes, de crecimiento lento, que suelen afectar a adultos varones, y que se localizan preferentemente en la cabeza y en el cuello. Aquellos tumores localizados en los miembros parecen ser más propensos a tener rasgos anatomopatológicos atípicos ${ }^{6}$, sin que se haya demostrado hasta el momento que este hecho 


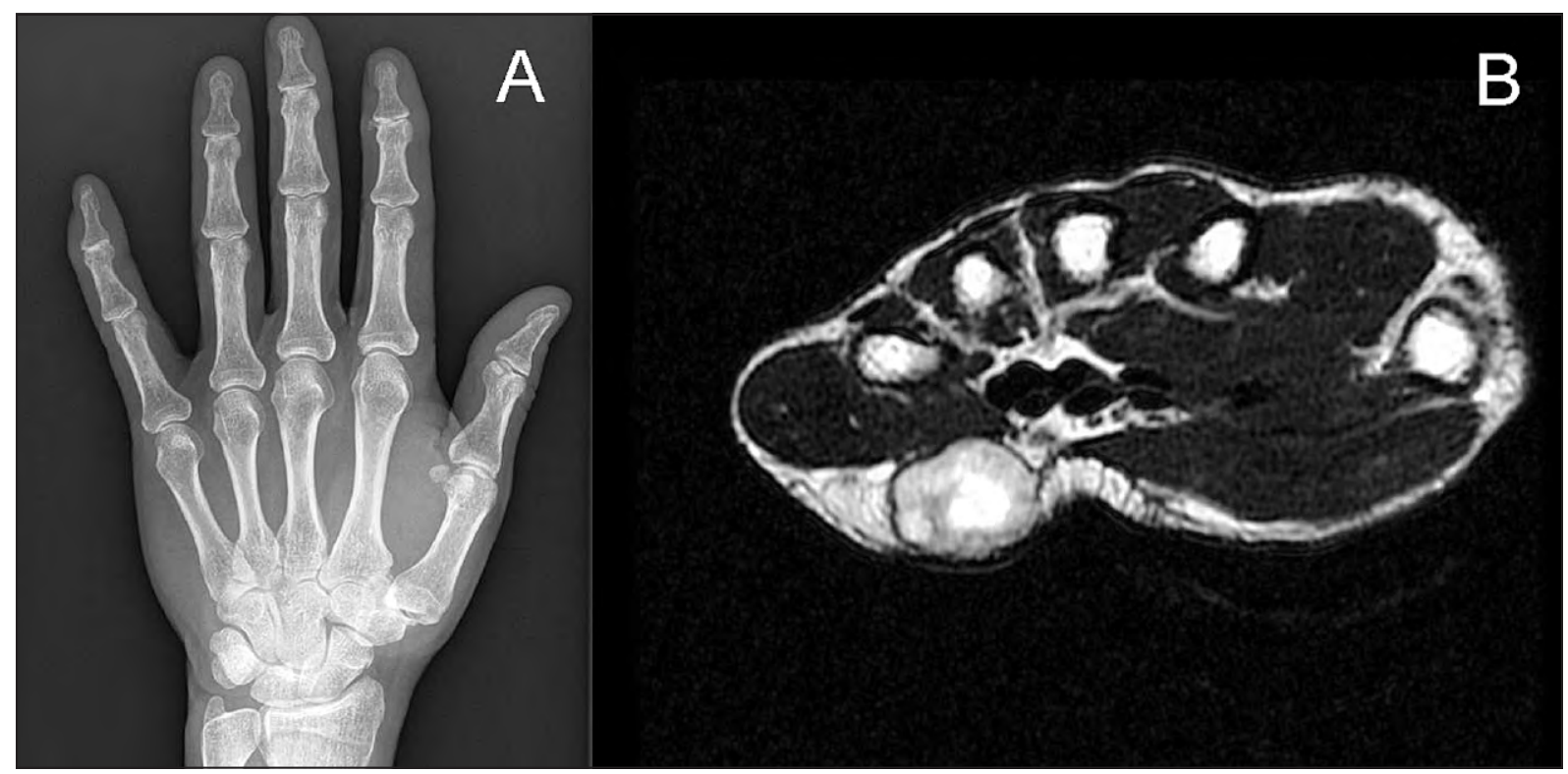

Figura 1. a) Radiografía AP sin alteraciones patológicas. b) Secuencia T2 Axial de RM con imagen de masa en región hipotenar de $2 \times 2 \mathrm{~cm}$, en relación con el paquete vasculonervioso cubital, sin características de agresividad local.

tenga una correlación con un comportamiento biológico más agresivo ${ }^{7}$.

\section{CASO CLÍNICO}

Paciente varón, de 58 años, diestro, con tumoración en la región hipotenar de la mano izquierda, de aproximadamente 3 años de evolución, sin antecedente traumático, que había aumentado de tamaño lentamente, no dolorosa y sin clínica de parestesias.

A la exploración, el paciente presentaba una tumoración de $3 \times 2 \mathrm{cms}$, de consistencia elástica, móvil, no adherida a planos profundos, sin evidencia de fístulas ni lesiones en la piel, no dolorosa y con Tinel negativo.

Se realizaron pruebas complementarias, no evidenciándose ninguna imagen patológica en la radiología simple (Figura 1a) y completándose el estudio con resonancia magnética que informó de un nódulo sólido en el tejido celular subcutáneo, hipointenso en secuencias potenciadas en T1 e hiperintenso aunque más heterogéneo en secuencias potenciadas en T2 (Figura 1b) y Stir. La lesión presentaba unos contornos bien definidos por una cápsula gruesa. Las dimensiones aproximadas eran de $2 \times 2 \times 1,5 \mathrm{~cm}$. Tras la administración de contraste intravenoso, se observaba una captación intensa de la cápsula, con escasa captación interna. Por su localización se encontraba en relación con la aponeurosis del flexor carpi radialis y la aponeurosis palmar y también con el paquete vasculonervioso cubital, por lo que la lesión probablemente tenía un origen mesenquimal (fibroma, neurinoma...), sin características de agresividad local.

Se realizó la extirpación quirúrgica, mediante un abordaje en $\mathrm{S}$ itálica, comprobándose la existencia de una tumoración única encapsulada, de consistencia elástica, blanca nacarada, que se encontraba en íntimo contacto con el paquete vasculonervioso cubital (Figura 2a).

El estudio anatomopatológico evidenció la presencia de una tumoración bien delimitada, con abundantes células de tipo epitelial y mioepitelial, citoplasma hialino, y un estroma condromixoide con estructuras seudoglandulares; todo ello compatible con el diagnóstico de SC (Figura 2b).

La evolución postoperatoria fue favorable, sin complicaciones en la cicatrización, ni problemas infecciosos, con movilidad distal completa y sin alteraciones sensitivas. Además, no han existido signos de recidiva al año de la intervención quirúrgica. 


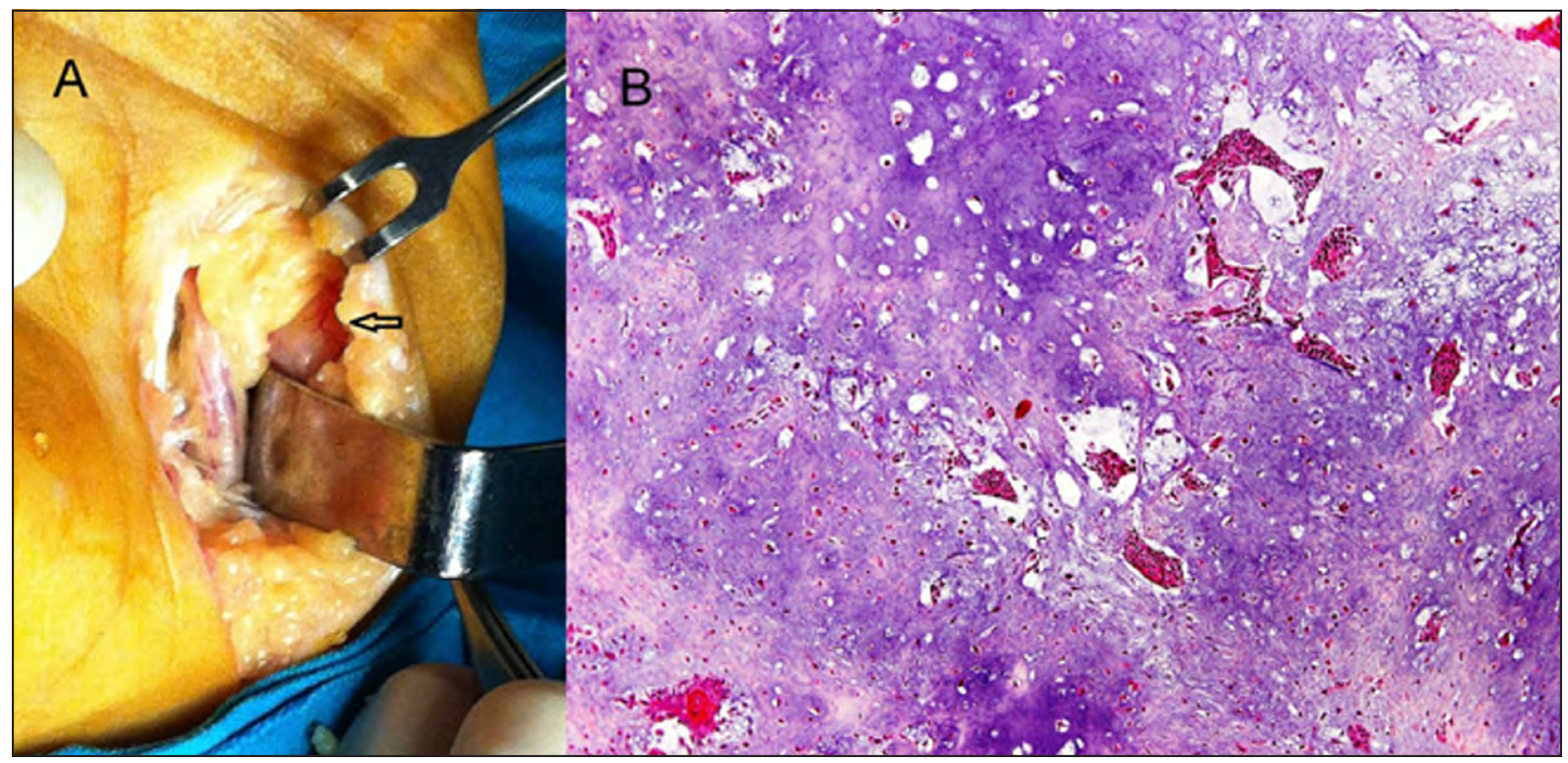

Figura 2. a) Imagen intraoperatoria. Lesión encapsulada, sin signos de agresividad, próxima al paquete vasculonervioso. b) Imagen microscópica (Tinción H-E, 10x). Abundantes células de tipo epitelial y mioepitelial, citoplasma hialino, y un estroma condromixoide con estructuras seudoglandulares; diagnóstico de siringoma condroide.

\section{DISCUSIÓN}

El SC, es un raro tumor cutáneo que se localiza preferentemente en la cabeza y en el cuello, a modo de una pequeña tumoración indolora de crecimiento lento, que comparte características microarquitecturales de doble origen mesenquimal y epitelial. En la mano es extremadamente rara, Terrill et al. ${ }^{8}$ reportaron dos casos en la mano; una en el dorso y otra en la palma, Ohtsuka y Tokuyama ${ }^{9}$ describió un caso en la región hipotenar. Haciendo una revisión de la bibliografía, se han encontrado veintitrés casos de siringomas condroides en la mano ${ }^{10}$, siendo el caso publicado por Beckler et al. ${ }^{11}$ el más parecido por localización al caso que presentamos.

El diagnóstico microscópico de estas lesiones es de certeza, apreciándose estructuras tubuloalveolares y de tipo glandular con dos o más capas de células cuboidales, poligonales, dispuestas a modo de islas en el seno de un estroma fibroadiposo, condroide, mixoide o hialino. En el diagnóstico diferencial histológico se deben considerar las siguientes entidades: condrosarcoma mixoide extraesquelético, paracordoma, neurofibromas y el tumor fibromixoide y osificante de partes blandas.
El tratamiento definitivo de estos tumores es quirúrgico, existiendo posibles recurrencias cuando no se realiza la extirpación completa.

Como regla general se trata de un tumor benigno, si bien la transformación maligna es un hecho posible. Para ello se han propuesto distintos criterios histológicos ${ }^{6}$, de entre los cuales, la atipia celular, el índice mitótico y la invasión local parecen ser los más importantes. No obstante, la recurrencia local y, sobre todo, la diseminación a distancia son los criterios inequívocos más importantes de malignidad. Por ello, algunos autores han propuesto el término de tumor mixto cutáneo atípico para aquellos siringomas condroides con rasgos histológicos de malignidad, especialmente la invasión local, pero sin evidencia de metástasis, dado que los rasgos histológicos atípicos no siempre se han correspondido fidedignamente con un comportamiento maligno de este raro tumor anexial. El caso de Medina et al. ${ }^{12}$ ilustra las características de este tipo de tumores, el diámetro es por lo general de 2-10 cm y la lesión ha estado presente durante años por ser asintomática, siendo más frecuente en mujeres de más de 50 años.

Aunque el siringoma condroide raramente aparece en la mano, se debe incluir en el diagnóstico diferencial de los tumores blandos de la mano ${ }^{13}$. 


\section{BIBLIOGRAFÍA}

1. Billroth T. Beobachtungen iffier Geschwulste der Speicheldrüsen. Virchow Arch Path Anat, 1859; 17: 357-75.

2. Chen A, Moreano E, Houston B, Funk GF. Chondroid syringoma of the head and neck: clinical management and literature review. Ear Nose Throat J, 1996; 75: 104-8.

3. Hirsch P, Helwig EB. Chondroid syringoma. Mixed tumor of skin, salivary gland type. Arch Dermatol, 1961; 84: 835-47.

4. Headington JT. Mixed tumors of skin: eccrine and apocrine types. Arch Dermatol, 1961; 84: 989-96.

5. Sungur N, Uysal A, Gumus M, Kocer U. An unusual chondroid syringoma. Dermatol Surg, 2003; 29: 977-9.

6. Trown K, Henaan PJ. Malignant mixed tumor of the skin (malignant chondroid syringoma). Pathology, 1994; 26: 237-47.

7. Barnett MD, Wallack MK, Zuretti A, Mesia L, Emery RS, Berson AM. Recurrent malignant chondroid syringoma foot. Am J Clin Oncol, 2000; 23: 227-32.

8. Terrill RQ, Groves RJ, Cohen MB Two cases of chondroid syringoma of the hand. J Hand Surg Am, 1987; 12: 1094-7.

9. Ohtsuka H, Tokuyama M. Chondroid syringoma in the hypothenar region. Ann Plast Surg, 1986; 17: 165-8.
10. Borman $\mathrm{H}$, Murat L, Ertas $\mathrm{N}$, Arican U, Colak A. Chondroid syringoma of the hand. Scand J Plast Reconstr Surg Hand Surg, 2009; 43: 291-3.

11. Bekler H, Gokce A, Beyzadeoglu T. Chondroid Syringoma of the hand: A rare localization. Handchir Mikrochir Plast Chir, 2007; 39: 430-2.

12. Medina J, Navarro R, Nagel D, Foucher G. Malignant chondroid syringoma of the hand. Scand J Plast Reconstr Surg Hand Surg, 2001; 35: 437-9.

13. Nemoto $\mathrm{K}$, Kato $\mathrm{N}$, Arino $\mathrm{H}$. Chondroid syringoma of the hand. Scand J Plast Reconstr Surg Hand Surg, 2002; 36: 379-81. 